\title{
Reflexiones sobre la Declaración de Cancún 2008: el derecho a la nutrición en los hospitales
}

\author{
Reflections on the Cancun Declaration 2008: The Right to \\ Nutrition in Hospitals
}

\author{
Humberto Arenas-Márquez ${ }^{1 *}$
}

Recibido: 19 de julio de 2018. Aceptado para publicación: 17 de febrero de 2019

Publicado en línea, marzo 11 de 2019

https://doi.org/10.35454/rncm.v2n1.059

\section{Resumen}

Esta reflexión crítica analiza si lo expresado en la Declaración de Cancún hace 10 años ha repercutido en mejorar la calidad de atención nutricional intrahospitalaria en Latinoamérica.

La pregunta es: ¿el paciente recibe una nutrición óptima basada en criterios de calidad (oportunidad, eficiencia, efectividad y seguridad)? La respuesta basada en evidencias es un no "significativo".

Podemos dividir en dos grandes secciones las fallas que deterioran nutricionalmente a los pacientes; las denominadas fallas latentes que son fallas en el sistema y las activas que son aquellas realizadas por las diversas personas que actúan en forma directa en la atención del paciente. Por lo tanto, las acciones para mejorar la seguridad nutricional del paciente hospitalizado deberán estar enfocadas en el cambio proactivo y preventivo, tanto en lo sistémico (sistema) como en lo individual (personas). Estas acciones podrán ser reguladas o auto-reguladas; sin embargo, nuestra visión es que la seguridad nutricional en el sistema de salud no tendrá significado sin la colaboración y compromiso de los profesionales de la medicina. Un cambio cultural es una precondición para enfocarnos en las acciones con el propósito de mejorar la seguridad en la atención nutricional del paciente, y también en una política de resultados. Hay que poner en la agenda política la atención nutricional segura del paciente. Encontrar en forma simultánea calidad de atención nutricional, eficiencia operacional e incentivo financiero ligado al resultado debe ser la meta. Tratar de resolver independientemente el problema es pérdida de tiempo y de esfuerzo.

Palabras clave: seguridad, calidad, cultura.

\section{Summary}

Overview: Ten years after the Declaration of Cancun, I am doing a critical analysis to evaluate if what was expressed in it has had repercussions on the improvement of the quality of inhospital nutritional care in Latin America.

The question is: Does the patient receive optimal nutrition based on quality criteria (timeliness, efficiency, effectiveness and safety)? The evidence-based answer is a "significant" no. We can divide in 2 big sections the failures that nutritionally damage patients; the so-called latent failures that are failures in the system and the active ones that are those made by the different people that act directly on the patient's attention. Therefore, actions to improve the nutritional security of the hospitalized patient should be focused on proactive and preventive change at both the systemic (system) and individual (people) levels. These actions may be regulated or self-regulated; however, our vision is that nutritional security in the health system will mean nothing without the collaboration and commitment of medical professionals. A cultural change is a precondition for focusing on actions to improve the safety of the patient's nutritional care and also on a policy of outcomes. Secure patient nutrition care must be put on the political agenda. Simultaneously finding quality of nutritional care, operational efficiency and financial incentive linked to the outcome must be the goal. Trying to solve the problem independently is a waste of time and effort.

Keywords: Security; Quality; Culture. 
A 10 años de la Declaración de Cancún debemos hacer un análisis crítico para evaluar si lo expresado en ella ha repercutido en una mejora de la calidad de atención nutricional intrahospitalaria especialmente en Latinoamérica.

Para iniciar esta reflexión me referiré a la medicina hipocrática la cual fue considerada ciencia y arte por el imperativo de modificar y adaptar la dieta del paciente enfermo de acuerdo con su condición para evitar el sufrimiento y la muerte.

Por otro lado, el Principio Hipocrático de "Primero no Dañar” está ahora más vigente que nunca, especialmente en la arena de la nutrición clínica y la terapia nutricional ${ }^{(1,2)}$.

Sin duda a 50 años del inicio de la Nutrición Clínica, tecnológicamente hemos presumido de grandes avances; a pesar de ello, en el Siglo XXI podemos afirmar que al menos en el ámbito intrahospitalario el paciente recibe alimentación, pero la pregunta relevante es si esta cubre los criterios de calidad: oportunidad, eficiencia, efectividad y seguridad.

La respuesta basada en evidencias es un no "significativo" ${ }^{3,4)}$.

Así pues podemos dividir en dos grandes secciones las fallas que deterioran nutricionalmente a los pacientes; las denominadas latentes que son fallas en el sistema y las activas que son aquellas realizadas por las diversas personas que actúan en forma directa en la atención del paciente. Por lo tanto, las acciones para mejorar la seguridad nutricional del paciente hospitalizado deberán ser enfocadas en el cambio proactivo y preventivo tanto en el nivel sistémico (sistema) como en el individual (personas). Estas acciones podrán ser reguladas o auto-reguladas; sin embargo, nuestra visión es que la seguridad nutricional en el sistema de salud no tendrá significado sin la colaboración y compromiso de los profesionales de la medicina.

Un cambio cultural es una precondición para la acción $y$ es un componente integral que conduce a acciones para mejorar la seguridad en la atención nutricional del paciente, $\mathrm{y}$ también a una política de resultados.

El cambio cultural es fundamental para la absorción de cualquier forma de innovación en atención para la salud.

La cultura es el elemento más importante en política. Por tanto, se requiere un cambio cultural mayor, especialmente en nuestro país, en áreas fundamentales como son: confiar en lo que hacemos bien, reportar lo que nos sale bien, pero también lo que nos sale mal, ser transparentes en nuestros resultados y disciplinados en perseguir la meta de la óptima nutrición para todos los pacientes bajo todas las circunstancias ${ }^{(5)}$.

Existen seis diferentes clases de cultura relacionadas con acciones para mejorar la seguridad en la atención nutricional del paciente.

1. La cultura de los profesionales (médicos, enfermeras, nutricionistas y farmacéuticos). Un cambio cultural es necesario para mejorar la confianza entre los profesionales y el paciente en términos de actitudes de los profesionales y sus creencias acerca de la atención nutricional proporcionada y cómo compartir la responsabilidad de dicha atención. Una información clara de que los médicos poco sabemos de nutrición clínica es fundamental para crear una relación más justa entre médicos, nutricionistas y pacientes, y un mejor entendimiento de las expectativas de unos y otros. Como resultado de este cambio cultural mediado por la información, la sociedad tendrá un gran beneficio ${ }^{(6)}$.

2. La cultura de los líderes en el sistema de salud y en los directores de hospitales debe ser cambiada para mejorar la seguridad nutricional del paciente. Existe, en general, poca cooperación entre los líderes y los que proporcionan la atención nutricional. La cultura de la colaboración profesional es clave para implementar de manera adecuada medidas de seguridad nutricional centradas en el paciente.

3. La cultura libre de culpabilidad. A los profesionales debe asegurárseles que los reportes realizados no serán causa de castigo, no tendrán carácter punitivo.

4. La cultura de la atención nutricional segura crea colaboración y confianza, y esos factores crearán una disminución en los costos.

5. La cultura de la atención nutricional centrada en el paciente dependerá del compromiso del paciente en promover su autocuidado y de la transparencia de la relación entre el paciente y el médico. Esto llevará a un cambio cultural en la atención nutricional de los proveedores al enfocarse en acciones por la nutrición segura del paciente con el fin de maximizar un impacto positivo, tanto desde el punto de vista social como económico.

6. Finalmente, el impacto social de estas acciones políticas para mejorar la seguridad en la atención nutricional del paciente serán influenciadas por algún grado de cultura individual del mismo.

La seguridad en la atención nutricional del paciente es de alta prioridad en la agenda política de muchos países, sin embargo, la naturaleza política del mismo punto 
puede presentar barreras para evaluar el impacto de las acciones. Hay que poner en la agenda política la atención nutricional segura del paciente.

Con el objetivo de mejorar la seguridad y la calidad en la atención nutricional deberíamos:

1. Estandarizar los procesos de atención nutricional que garanticen que los pacientes reciben la óptima atención nutricional.

2. Construir la infraestructura correcta; equivale a contar con un servicio de atención de alimentación óptima, y solo cuando este objetivo no pueda ser satisfecho por razones inherentes al paciente, contar con un cuadro básico de nutrición artificial pero que sea indicada, monitorizada y gestionada por el equipo humano correcto.

3. Documentar con datos duros el resultado de la aplicación excelente de la terapia, que permita demostrar el verdadero impacto económico de una terapia nutricional óptima.

4. Verificar, mediante una acreditación óptima, aquellos centros que proporcionen excelencia en dicha atención nutricional. Lo que permitiría incentivar a los que lo hacen mejor, y a la vez estandarizar e imitar los procesos que llevan a esa excelencia de atención nutricional ${ }^{(7)}$.

Enfrentar esos retos requiere coraje y liderazgo. La meta debe ser encontrar en forma simultánea calidad de atención nutricional, eficiencia operacional e incentivo financiero ligado al desempeño ${ }^{(8-10)}$. Tratar de resolver independientemente el problema es perder el tiempo y el esfuerzo. Caminemos pues, basados en la Declaración de Cartagena de las palabras a las acciones bajo propuestas que cubran estos objetivos.

\section{Financiación}

El presente artículo no tuvo financiación.

\section{Conflicto de intereses}

El autor declara no tener conflicto de intereses.

\section{Referencias bibliográficas}

1. Cárdenas D, Pelluchon C. The Ethical Foundations of Nutritional Care: A viewpoint on the recent ESPEN guidelines. Clin Nutr. 2016; 35(6):1587-8.

2. Correia MI, Waitzberg D. The impact of malnutrition on morbidity, mortality, length of hospital stay and cost evaluated though a multivariate model analysis. Clin Nutr. 2003;22(3):235-9.

3. Correia MI, Hegazi RA. Addressing Disease-Related Malnutrition in Helathcare: A Latin American Perspective. JPEN J Parenter Enter Nutr. 2016;40(3):319-25.

4. Correia MI. Perman M. Waitzberg D. Hospital Malnutrition in Latin America: A systematic review. Clin Nutr. 2017;36 (4):958-67.

5. Arenas $\mathrm{H}$. El futuro de la seguridad del paciente y la calidad en la atención médica. En: Pérez JA, editor. Alfil. Seguridad del paciente al alcance de todos: CDMX; 2013. p. 495-15.

6. Arenas H. Educación en Nutrición Clínica. Rev Nutr Clin Metab. 2018;1(2):13-16.

7. Hoyt BD. Looking forward. Bull Am Coll Surg. 2011;96(1):4-5.

8. Tapenden KA. The Value of Nutrition Intervention. JPEN J of Parent Ent Nut. 2013;37(2):160.

9. Klek S, Chourdakis M, Bischoff S, Dubrow S, Forbes A, et al. Economy matters to fight against malnutrition: Results from a multicenter survey. Clin Nutr. 2017;36: 162-9.

10. Correia I, Perman M, Pradelli L, Omaral S, Waitzberg DL- J. Economic burden of Hospital Malnutrition and the cost benefit of supplemental Parenteral Nutrition in critically ill patients in Latin America. Med Econ. 2018;13:1-23. 